Proceedings of the 1999 Particle Accelerator Conference, New York, 1999

\title{
MAGNETIC FIELD CALCULATION FOR A 13 MEV PET CYCLOTRON*
}

\author{
W.Y. Yang, M. Yoon , S. Oh, Dept. of Physics, POSTECH, Korea, \\ J.S.Chai, J.H. Ha, KCCH, Korea
}

\begin{abstract}
A design study of the main magnet for a $13 \mathrm{MeV}$ cyclotron has been carried out in a joint collaboration between the Korea Cancer Center Hospital(KCCH) and POSTECH. A maximum energy of $13 \mathrm{MeV}$ has been chosen to produce radio-isotopes such as ${ }^{18} \mathrm{~F}$. There are four magnet sectors, each with radial-ridged shape. Maximum magnetic fields are $1.85 \mathrm{~T}$ and $0.48 \mathrm{~T}$ at hill and valley centers, respectively. The total size of the cyclotron is less than $2 \mathrm{~m}$ in diameter. The program TOSCA has been utilized for the field calculation and optimization. In this paper, we describe design parameters of the $13 \mathrm{MeV}$ PET cyclotron, with emphasis on the magnetic field shape and the beam optics calculation.
\end{abstract}

\section{INTRODUCTION}

At the Korea Cancer Center Hospital (KCCH), design study of a $13 \mathrm{MeV}$ cyclotron for Positron Emission Tomography (PET) has been in progress since 1997. The study has been carried out in a joint collaboration between $\mathrm{KCCH}$ and the Pohang University of Science and Technology (POSTECH).

The KCCH has played a leading role in Korea in radiation medicine as well as in the treatment and research of cancer, since it was established as the Radiological Research Institute in 1963 to promote the medical application of atomic energy in Korea. For neutron therapy and radiation treatment, a $50 \mathrm{MeV}$ medical cyclotron, built by Scanditronix, was installed at the hospital in 1986. The cyclotron has provided an in-house source of radio-isotopes such as ${ }^{201} \mathrm{Tl}$, ${ }^{123} \mathrm{I},{ }^{67} \mathrm{Ga}$, etc, and in particular, the shorter-lived radioisotopes for diagnostic or clinical use. In addition to serving in-house duties, this cyclotron has also produced and supplied $15 \%$ of all cyclotron based radio-isotopes in Korea. This service has greatly contributed towards awareness of the potential benefits of nuclear medicine afforded by particle accelerators and evoked calls for similar services in other hospitals in Korea. So far only two hospitals have installed dedicated cyclotrons for PET (Positron Emission Tomography) applications, where the isotopes of interest are the four clinically significant positron emitters ${ }^{15} \mathrm{O},{ }^{13} \mathrm{~N},{ }^{11} \mathrm{C}$, and ${ }^{18} \mathrm{~F}$ in particular.

At the $\mathrm{KCCH}$, increasing desire for an uninterrupted, reliable and timely supply of the isotopes to customers has prompted obtaining a dedicated 5-13 MeV cyclotron for PET applications and pursuing the purchase of another 30 $\mathrm{MeV}$ medical cyclotron in the very near future. A decision has been made to design the PET cyclotron in Korea. This

This work is supported by Ministry of Science and Technology of Korean government

Email: moohyun@postech.ac.kr will not only ease the problems associated with maintenance during operation but also keeps the door open for continuous upgrading of the machine in the future. The project is supported by the Ministry of Science and Technology (MOST) of the government, as a part of the $2^{\text {nd }}$ phase of the mid- and long-term nuclear energy research plan. The cyclotron construction project was officially started in April 1999 and is to be completed in three years.

In this paper, we introduce the major parameters of this $13 \mathrm{MeV}$ PET cyclotron with particular emphasis on the magnetic field design and the equilibrium orbit calculation.

\section{PARAMETERS}

Table I shows major design parameters for the $13 \mathrm{MeV}$ PET cyclotron. Maximum beam energy was chosen to be $13 \mathrm{MeV}$ which is above the threshold energy for ${ }^{18} \mathrm{~F}$ production. A four-sector magnet structure is employed with radial-ridged shape. Hill angle is chosen to be 46 degree, but the effective hill angle seen by the beam increases slightly in radius because the vertex of the hill is not at the center of the cyclotron, as will be described later. For ion source, internal PIG source will be used. Though this will deteriorate the vacuum in the central region, it is more economical and simpler than adopting an external ion source. In order to provide an efficient vacuum conducting path in the central region of the cyclotron, there are four holes in the valley region.

Negatively charged hydrogen ions will be used for acceleration because extraction is much easier than for positively charged ions. Accelerating negative ions has also an advantage in that the maximum extracted energy can be varied easily by simply repositioning the stripping foil.

Magnetic fields at $13 \mathrm{MeV}$ are $1.85 \mathrm{~T}$ and $0.48 \mathrm{~T}$ at the hill and valley centers, respectively. Maximum average magnetic field is about 1.2 T. Harmonic number is four, and therefore the radio frequency is about $72 \mathrm{MHz}$. Dee voltage is $40 \mathrm{kV}$. The energy gain per turn is given by

$$
\Delta E=4 q V_{d e e} \sin \frac{h \theta_{d e e}}{2}
$$

where $V_{\text {dee }}$ is the dee voltage, $h$ is the harmonic number and $\theta_{d e e}$ is the dee angle. With $V_{d e e}=40 \mathrm{kV}, h=4, \theta_{d e e}=43.6^{\circ}$, the energy gain per turn is $\Delta E=159 \mathrm{kV}$. The total number of turns to reach $13 \mathrm{MeV}$ is therefore approximately 82 . 
Table I : Main parameters of the $13 \mathrm{MeV}$ PET cyclotron

\begin{tabular}{|c|c|c|}
\hline Parameter & Unit & Value \\
\hline Maximum energy & $\mathrm{MeV}$ & 13 \\
\hline Beam species & & $\begin{array}{l}\text { Negative } \\
\text { hydrogen }\end{array}$ \\
\hline Number of sectors & & 4 \\
\hline Ion source & & $\begin{array}{c}\text { Internal } \\
\text { negative PIG }\end{array}$ \\
\hline Hill angle & degrees & 46.0 \\
\hline Valley angle & degrees & 44.0 \\
\hline $\begin{array}{l}\text { Maximum average } \\
\text { magnetic field }\end{array}$ & $\mathrm{T}$ & 1.2 \\
\hline Harmonic number & & 4 \\
\hline Radio-frequency & $\mathrm{MHz}$ & 72 \\
\hline $\begin{array}{l}\text { Maximum average radius } \\
\text { of a beam }\end{array}$ & $\mathrm{cm}$ & 43 \\
\hline $\begin{array}{l}\text { Maximum orbit distance } \\
\text { from the cyclotron center }\end{array}$ & $\mathrm{cm}$ & 45 \\
\hline $\begin{array}{l}\text { Maximum magnetic field } \\
\text { at the hill center }\end{array}$ & $\mathrm{T}$ & 1.85 \\
\hline $\begin{array}{l}\text { Maximum magnetic field } \\
\text { at the valley center }\end{array}$ & $\mathrm{T}$ & 0.48 \\
\hline Beam current & $\mu \mathrm{A}$ & $\sim 20$ \\
\hline
\end{tabular}

\section{MAGNET SYSTEM}

In the initial stage of the cyclotron design, major cyclotron parameters have been calculated by employing a simple first-order theory $[1,2]$ in beam optics, from which orbit radius, average magnetic field, fields at the hill and valley, focusing frequencies are determined as a function of beam energy. Fig. 1 shows the side view of the $13 \mathrm{MeV}$ cyclotron. It shows that the height is approximately $93 \mathrm{~cm}$ and the diameter is $187 \mathrm{~cm}$. For magnetic field calculation in the

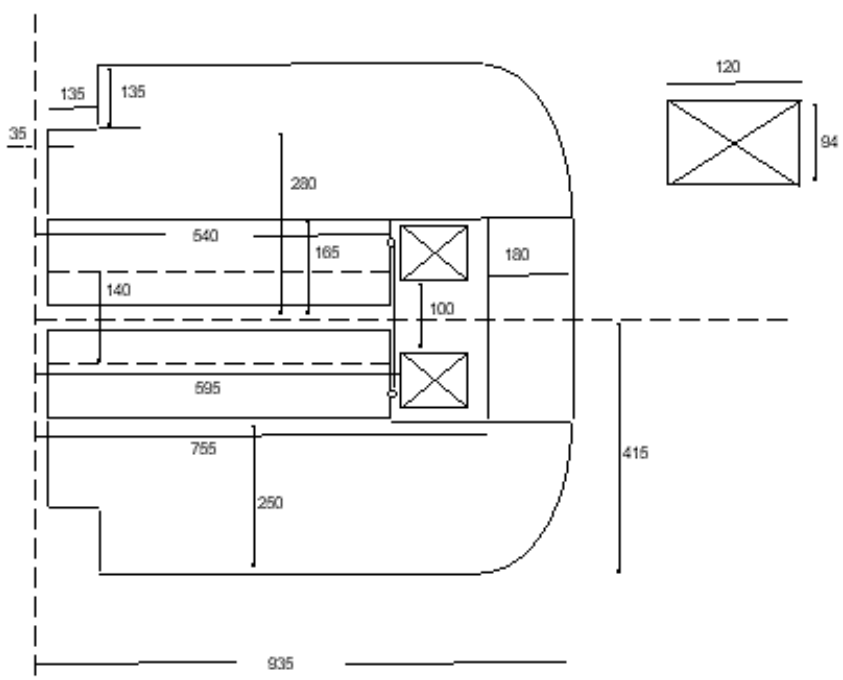

Figure 1: Side view of the $13 \mathrm{MeV}$ cyclotron

yoke and valley region, a two-dimensional program such as POISSON [3] has been utilized. Fig. 2 shows the twodimensional plot of the magnetic field line in the yoke and valley region, which is obtained with the help of POISSON program. Total current per each coil is 44,000 ampere-turns and the current density is about $3 \mathrm{~A} / \mathrm{mm}^{2}$.

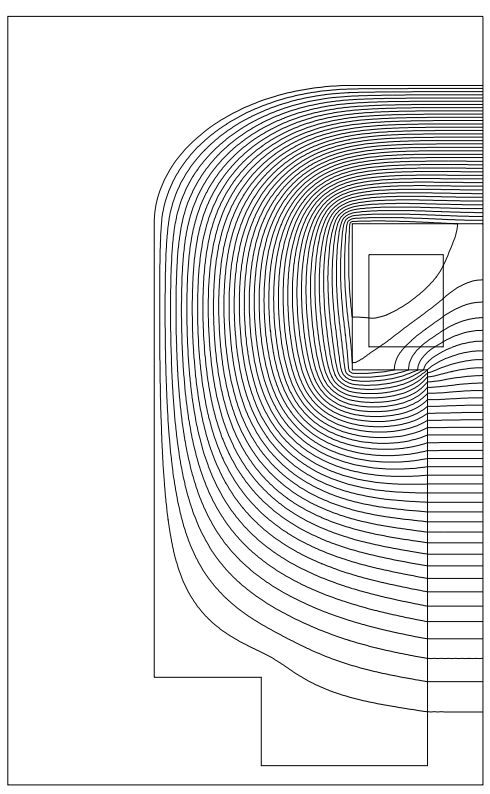

Figure 2: Magnetic field lines computed by POISSON

The OPERA-3D program was used to calculate the threedimensional magnetic field. Fig. 3 shows the geometry of the cyclotron modeled by the OPERA-3D program. This figure shows four holes at the valley center. Presence of these holes distorts the field greatly. A step by step optimization of the magnet geometry is thus needed.

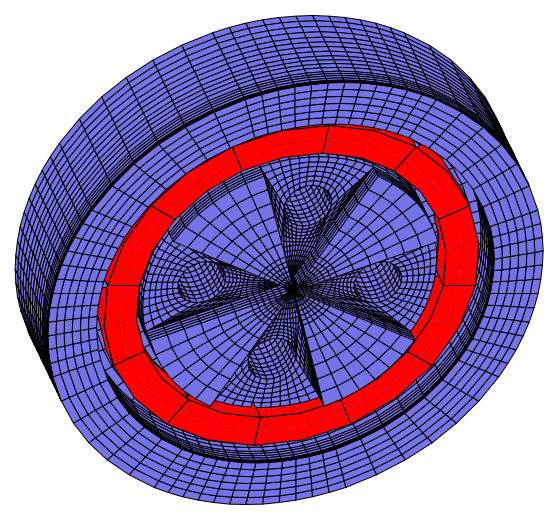

Figure 3: Cyclotron geometry modeled by OPERA-3D In order to maintain the isochronous field, we have em- 
ployed a variable vertical gap along the radius in the hill region, rather than using conventional trim coils. This makes the cyclotron operation much simpler, avoiding complicated optimization procedure with trim coils. Simplicity in operation is very important for hospital-based cyclotron where resources for technical support are limited. When the vertical hill gap decreases in steps along the radius, the average magnetic field seen by an accelerating beam is increased in radius. We have been able to obtain a reasonable isochronism by adjusting the hill gaps. Difference in gap height at the minimum and maximum radius is about $6 \mathrm{~mm}$. Fig. 4 shows the average magnetic field as a function of average beam radius. In this figure the average magnetic fields are the averaged fields along the equilibrium orbits. For given magnetic fields, equilibrium orbits are found by solving linearized equations of motion whose details will not be described here.

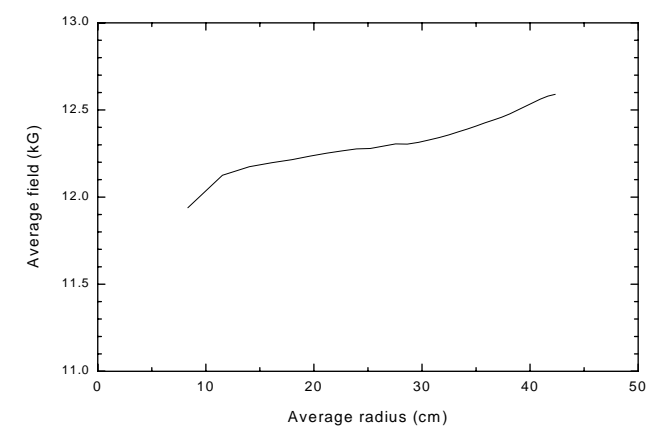

Figure 4: Average magnetic field for the $13 \mathrm{MeV}$ cyclotron as a function of radius

Fig. 5 shows the frequency error due to the magnetic field given in Fig. 4. The vertical axis of this figure indicates $\omega_{0} / \omega-1$, where $\omega_{0}$ is a fixed frequency which is basically the radio frequency and $\omega$ is the angular revolution frequency of a particle. From this figure, one can obtain the phase error of the particle using

$$
\sin \phi(E)=\sin \phi_{i}+\frac{2 \pi h}{q V} \int\left(\frac{\omega_{0}}{\omega}-1\right) d E
$$

where the integral extends from the initial energy $E_{i}$ where $\phi=\phi_{i}$ to the given energy $E$. With the above equation applied to Fig. 5, we obtain the total phase excursion is within $\pm 15^{\circ}$.

Horizontal and vertical focusing frequencies $\left(\nu_{r}\right.$ and $\left.\nu_{z}\right)$ are also obtained from the equilibrium orbit calculation. Fig. 6 describes the excursion of $\nu_{r}$ and $\nu_{z}$ from injection to extraction radius. The lines in this figure represent $2 \nu_{y}=1$, $\nu_{x}=3 \nu_{y}, \nu_{x}=1,3 \nu_{x}+\nu_{y}=4,3 \nu_{x}=4,3 \nu_{x}-\nu_{y}=-4$ resonances. Therefore these are the structure(or perfect) resonances arising from the 4-fold symmetry of the magnet. It is seen that focusing frequencies are sufficiently away from the resonances.

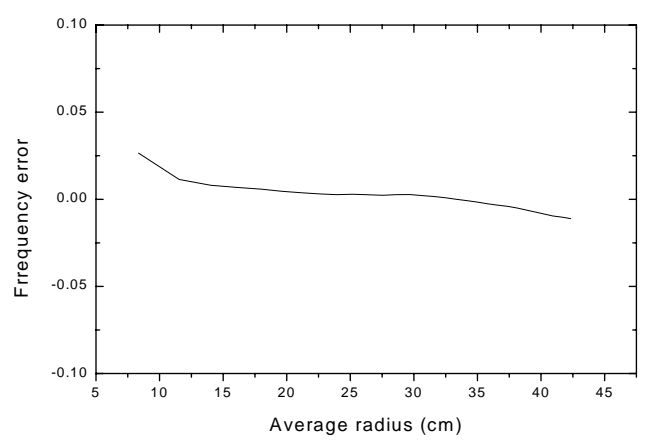

Figure 5: Frequency error as a function of radius for the $13 \mathrm{MeV}$ cycl

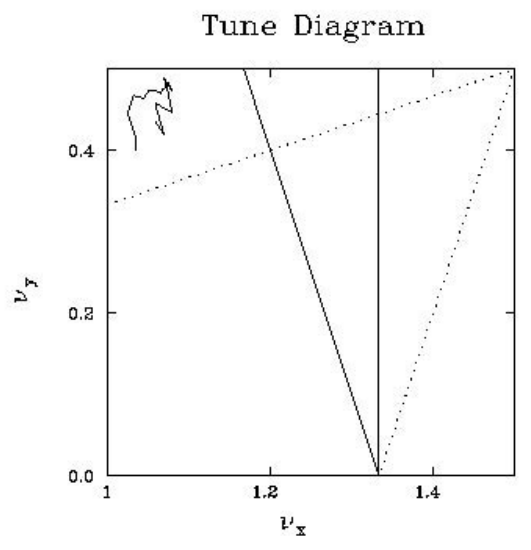

Figure 6: Tune diagram for the $13 \mathrm{MeV}$ cyclotron

\section{SUMMARY}

In Korea, design studies for a $13 \mathrm{MeV}$ PET cyclotron have been in progress. Currently, design of main magnets and the rf system are being carried out. When completed in 2002 , this cyclotron will serve to produce short-lived radioisotopes like ${ }^{18} \mathrm{~F}$.

\section{REFERENCES}

[1] M. Yoon, J.S.Chai, J.W.Kim, W.Y.Yang and S.Oh, Proceedings of the International Cyclotron Conference, Caen, France, 1998

[2] S. Oh, W.Y. Yang, M. Yoon, J.S.Chai, J.H. Ha, J.W. Kim, Proceedings of the European Particle Accelerator Conference, Stockholm, Sweden, 1998

[3] User's Guide for the POISSON/SUPERFISH Group of Codes, LA-UR-87-115, Los Alamos Accelerator Code Group 